\title{
Prognostic significance of receptor expression discordance between primary and recurrent breast cancers: a meta-analysis
}

\author{
Sho Shiino ${ }^{1,2} \cdot$ Graham Ball $^{3}$ - Binafsha M. Syed ${ }^{4} \cdot$ Sasagu Kurozumi ${ }^{5,6} \cdot$ Andrew R. Green $^{1} \cdot$ Hitoshi Tsuda ${ }^{7}$. \\ Shin Takayama ${ }^{2} \cdot$ Akihiko Suto $^{2} \cdot$ Emad A. Rakha $^{1,8}[$
}

Received: 3 March 2021 / Accepted: 6 September 2021 / Published online: 6 October 2021

(c) The Author(s) 2021

\begin{abstract}
Purpose This meta-analysis aimed to investigate whether receptor (estrogen receptor [ER], progesterone receptor [PR], and human epidermal growth factor receptor 2 [HER2]) discordances between primary and recurrent breast cancers affect patients' survival.

Methods Search terms contained ER, PR, and HER2 status details in both primary and recurrent tumors (local recurrence or distant metastasis) in addition to survival outcome data (overall survival [OS] or post-recurrence survival [PRS]).

Results Loss of ER or PR in recurrent tumors was significantly associated with shorter OS as compared with receptorpositive concordance (hazard ratio [HR], 1.67; 95\% confidence interval [\% CI] 1.37-2.04; $p<0.00001$ and HR, $1.45 ; 95 \%$ CI $1.21-1.75 ; p<0.0001$, respectively). Similar trends were observed in groups with only distant metastasis. Gain of ER was a significant predictor of longer PRS as compared with receptor-negative concordance (HR, 0.76; 95\% CI 0.59-0.97; $p=0.03$ ). Gain of PR was not a significant predictor of longer survival compared with receptor-negative concordance, but it could be related to better OS at distant metastasis. Both HER2 of loss and gain could be related to poor outcomes.

Conclusion This meta-analysis showed that receptor conversion in recurrent tumors may affect patient survival as compared with receptor concordance.
\end{abstract}

Keywords Breast cancer $\cdot$ Receptor discordance $\cdot$ Estrogen receptor $\cdot$ Progesterone receptor $\cdot$ Human epidermal growth factor receptor $2 \cdot$ Prognosis

$\begin{array}{ll}\text { Abbreviations } \\ \text { ER } & \text { Estrogen receptor } \\ \text { PR } & \text { Progesterone receptor } \\ \text { HER2 } & \text { Human epidermal growth factor receptor 2 } \\ \text { BC } & \text { Breast cancer } \\ \text { PRISMA-P } & \begin{array}{l}\text { Preferred reporting items for systematic } \\ \text { review and meta-analysis protocols }\end{array}\end{array}$

Emad A. Rakha

mrzear1@exmail.nottingham.ac.uk

1 Division of Cancer and Stem Cells, School of Medicine, University of Nottingham, Nottingham, UK

2 Department of Breast Surgery, National Cancer Center Hospital, Tokyo, Japan

3 School of Science \& Technology, John Van Geest Cancer Research Centre, Nottingham Trent University, Clifton Campus, Clifton Lane, Nottingham, UK

4 Head of Clinical Research Division, Medical Research Centre, Liaquat University of Medical \& Health Sciences, Jamshoro, Pakistan
PICO Participants, interventions, comparators, and outcomes

PROSPERO A prospective international register of systematic reviews

TNM Tumor-Node-Metastasis

IHC Immunohistochemical

5 Department of Breast Surgery, International University of Health and Welfare, Narita, Japan

6 Department of General Surgical Science, Gunma University Graduate School of Medicine, Maebashi, Japan

7 Department of Basic Pathology, National Defense Medical College Hospital, Tokorozawa, Japan

8 Department of Histopathology, Nottingham University Hospital NHS Trust, City Hospital Campus, Hucknall Road, Nottingham NG5 1PB, UK 


\begin{tabular}{|c|c|}
\hline ESMO & $\begin{array}{l}\text { The European Society for Medical } \\
\text { Oncology }\end{array}$ \\
\hline ASCO/CAP & $\begin{array}{l}\text { American Society of Clinical Oncology/ } \\
\text { College of American Pathologists }\end{array}$ \\
\hline RoBANS & $\begin{array}{l}\text { The Risk of Bias Assessment tool for Non } \\
\text { randomized Studies }\end{array}$ \\
\hline HR & Hazard ratio \\
\hline CI & Confidence interval \\
\hline FNA & Fine-needle aspiration \\
\hline OS & Overall survival \\
\hline PRS & Post-recurrence survival \\
\hline
\end{tabular}

\section{Introduction}

Hormone receptors (estrogen receptor [ER] and progesterone receptor $[\mathrm{PR}]$ ) and human epidermal growth factor receptor 2 [HER2] status in primary breast cancers (BC) not only provides prognostic information but it is also crucial for deciding on an effective treatment plan. Previous studies have reported discordance in these three biological markers between primary and recurrent tumors [1-4].

Some meta-analyses have clarified the rates of receptor discordance in these biological markers between primary and recurrent tumors $[5,6]$. In a recent meta-analysis, ER, PR, and HER2 receptor discordance rates were reported as 19.3\% (95\% CI 15.8-23.4), 30.9\% (95\% CI 26.6-35.6), and $10.3 \%$ (95\% CI 7.8-13.6), respectively [5]. Current guidelines recommend that patients with $\mathrm{BC}$ metastasis undergo biopsy or excisional biopsy to evaluate the status of these receptors $[7,8]$.

Previous studies have reported that receptor discordance may affect patient survival [9-11]. By contrast, there is no survival difference between patients with receptor discordance and those with receptor concordance [2]. Thus, no clear consensus on whether discordances of these receptors affect patient survival has been reached. Moreover, it remains unknown whether adjuvant therapy can affect the survival of patients with receptor discordance $[5,10,11]$. The aim of this study is to investigate whether discordance of receptors (ER, PR, and HER2) between primary and recurrent tumors affect the survival of patients with BC compared with receptor concordance using a meta-analytic approach.

\section{Methods}

See Supplementary Text in the supplementary file for more information.

\section{Search strategy and eligibility criteria of articles}

We used preferred reporting items for systematic review and meta-analysis protocols (PRISMA-P, 2015) [12] to ensure a transparent and complete reporting of this research (Supplementary Table S1). The PICO elements (participants, interventions, comparators, and outcomes) for the clinical question, primary/secondary endpoints, inclusion/exclusion criteria, and subgroup analysis are shown in Supplementary Table S2. After searching the three electronic databases (MEDLINE, Cochrane library, and EMBASE), we excluded duplicated articles (Fig. 1).

A first screening was performed by reading the titles or abstracts. Studies meeting the following criteria were excluded in the first screening: (1) not relevant to our research objectives, (2) full title or abstract was unavailable, (3) gray literature (e.g., conference meeting abstracts, nonpeer-reviewed literature), (4) review article, (5) case report, or (6) no abstract in English. A second screening was performed based on a full-text review of each article. In the second screening, we excluded articles that were unrelated to our topic, contained data unavailable for further statistical analysis, or the full text was unavailable.

All hits from the three databases were independently evaluated for selection or exclusion by two authors (SS and BMS) (Fig. 1 and Supplementary Fig. S1). Discrepancies of articles were discussed until consensus was reached and the finally selected articles were decided by the 1st reviewer (SS). Any discrepancies of selected articles between the two independent reviewers were evaluated using the kappa $(\kappa)$ coefficient.

\section{Data extraction}

We extracted some information from the finally selected articles as shown in Supplementary Table S2. We sent emails to each corresponding author of the final selected articles to obtain sufficient data. We allowed use of these missing data in the peer-reviewed literature for our meta-analysis, although these data were unpublished.

Tumors were pathologically classified based on the Tumor-Node-Metastasis (TNM) staging system [13]. ER and PR statuses for immunohistochemical (IHC) staining were considered positive if the cut-off was either $\geq 1 \%$ or $\geq 10 \%$ in reference to the European Society for Medical Oncology (ESMO) guidelines [14] or American Society of Clinical Oncology/College of American Pathologists (ASCO/CAP) guidelines [15, 16]. The HER2 expression level was mainly defined as positive according to the 2007 or 2013 ASCO/CAP guidelines [17, 18].

The definition of "receptor discordance" groups was defined as follows: 
Fig. 1 Flow chart of the selection procedure used for this meta-analysis. $*$ The details are shown in Supplementary Fig. S1

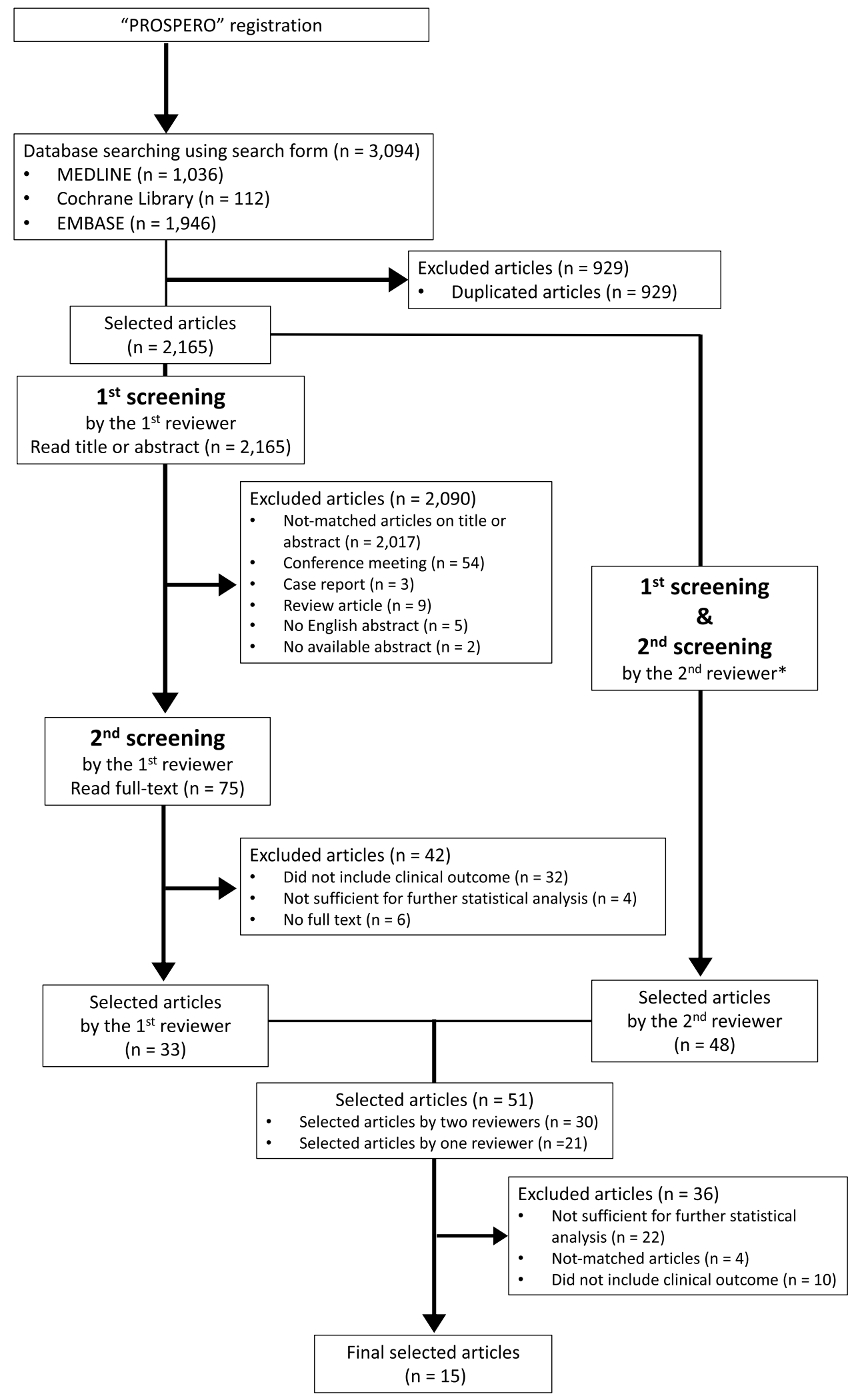

(1) receptor loss (positive to negative, +/-), status positivity with primary breast tumor converted to status negativity with recurrent breast tumor or
(2) receptor gain (negative to positive, $-/+$ ), status negativity with primary breast tumor converted to status positivity with recurrent breast tumor. 
Cases in which no change in status occurred between primary and recurrent tumors were considered to have "receptor concordance," which was either positive-to-positive $(+/+)$ receptor concordance or negative-to-negative (-/-) receptor concordance. We compared the differences in survival between receptor-discordant groups and receptor-concordant groups as follows: receptor-loss (+/-) group vs. receptorconcordant $(+/+)$ group or receptor-gain $(-/+)$ group vs. receptor-concordant $(-/-)$ group.

\section{Statistical analysis}

Hazard ratio (HR) and 95\% confidence interval (CI) were extracted from each study included in the final analysis. All results were calculated with a fixed-effects model. We also used a random-effects model to evaluate the difference in the heterogeneity between the two models. Statistical heterogeneity among studies was assessed using the $I^{2}$ test. We evaluated $I^{2}$ values according to the following definition: $I^{2}>50 \%$, high heterogeneity; $I^{2} 26-50 \%$, moderate heterogeneity; and $I^{2} 0-25 \%$, low heterogeneity.

Forest plots were used to visualize heterogeneity among studies. Funnel plots were made to evaluate publication bias. A two-sided $p$-value of $<0.05$ was considered statistically significant in both models. In cases where $I^{2}$ values were much greater than $50 \%$ or significant, we performed sensitivity analysis or meta-regression analysis to evaluate reasons for the high heterogeneity. The risk of bias graph and bias summary were performed using Review Manager (RevMan) version 5.3. (The Cochrane Collaboration, Oxford, England) [19].

In cases where HR data or survival data were not explicitly stated in the literature, we extracted cumulative survival values using Engauge Digitizer software v12 from the relevant Kaplan-Meier survival curves. Moreover, we estimated the HR values from the extracted cumulative survival values using the Microsoft Excel spreadsheet reported previously [20]. We excluded groups with either fewer than ten total cases or fewer than five total events from our meta-analysis because we could not calculate data using our methods when the total number of cases of either the receptor-concordant or receptor-discordant group was too few.

\section{Results}

\section{Characteristics of eligible studies}

We identified 2,165 articles after excluding duplicated articles from the three electrical databases. After the first and second screenings, a total of 15 studies met all inclusion criteria. The discordance rate of selected articles between the two reviewers was $1.0 \%(21 / 2,165)$, and the $\kappa$ value was 0.74 , indicating a fair to good agreement. Some studies $(4 / 15,26.7 \%)$ included Stage IV BC at primary diagnosis [11, 21-23]. We excluded these four articles from our metaanalysis to minimize selection bias because survival data of patients with Stage IV BC are likely to skew meta-analysis results. Thus, 11 studies were finally selected (Table 1 and Supplementary Table S4) [24-34]. In these studies, diagnosis of a metastatic lesion was mainly performed by either core-needle biopsy or surgical excision. Some studies (4/11, $36.4 \%$ ) included some cases that were diagnosed by fineneedle aspiration (FNA) of the metastatic lesion [25, 28, $30,31]$. We reasoned that these articles would not strongly affect survival data and therefore allowed their inclusion in our meta-analysis.

We evaluated these 11 remaining articles using the Risk of Bias Assessment tool for Non-randomized Studies (RoBANS) (Supplementary Text, Supplementary Fig. S2A and S2B). In each domain group, $\kappa$-values for the agreement between two reviewers were either excellent or fair to good as shown in Supplementary Fig. S2C. Of the 11 studies included, the number of articles that had performed overall survival (OS) analysis or post-recurrence survival (PRS) analysis was summarized in Supplementary Table S5.

Centralized laboratory testing was performed in 8 articles (8/11: 72.7\%). The details were as follows: 6 articles, testing for all samples; 1 article, testing for $45.6 \%$ of total cases (83/182 cases); and 1 article, testing for $84.3 \%$ of total cases (59/70). The median discordant rates for the receptor statuses were $19.7 \%$ (range, 10.7-32.5\%) for ER, 32.5\% (21.9-40.7\%) for PR, and 10.3\% (3.7-28.2\%) for HER2. The threshold definition of both ER and PR was either $\geq 1 \%$ $(4 / 11,36.4 \%)[24,27,29,34]$ or $\geq 10 \%(7 / 11,63.6 \%)$ [24-26, 28, 30, 31, 33]. Of these, one study investigated a survival difference effect on OS of simultaneously $\geq 10 \%$ and $\geq 1 \%$ [24]. Another study did not clearly mention the definition of hormone receptor positivity [32]. The positive definition for HER2/CEP17 ratio was mainly decided as either $\geq 2.2$ or $\geq 2.0$.

In the selected articles, survival data were available in six studies $(6 / 11,54.5 \%)$ each for (1) local recurrences and distant metastases [25, 28, 30-32, 34] or (2) only distant metastases [24-27, 29, 33]. One study investigated the survival difference of both (1) and (2) [25].

\section{Influence of receptor discordance on OS}

The ER-loss (+/-) and PR-loss (+/-) groups were significantly associated with shorter OS compared with the ERconcordant (+/+) and PR-concordant (+/+) groups, respectively ([Fixed-effects model] ER groups: HR, 1.67; 95\% CI 1.37-2.04; $p<0.00001$; PR groups: HR, $1.45 ; 95 \%$ CI 1.21-1.75; $p<0.0001$, [Random-effects model] ER groups: 


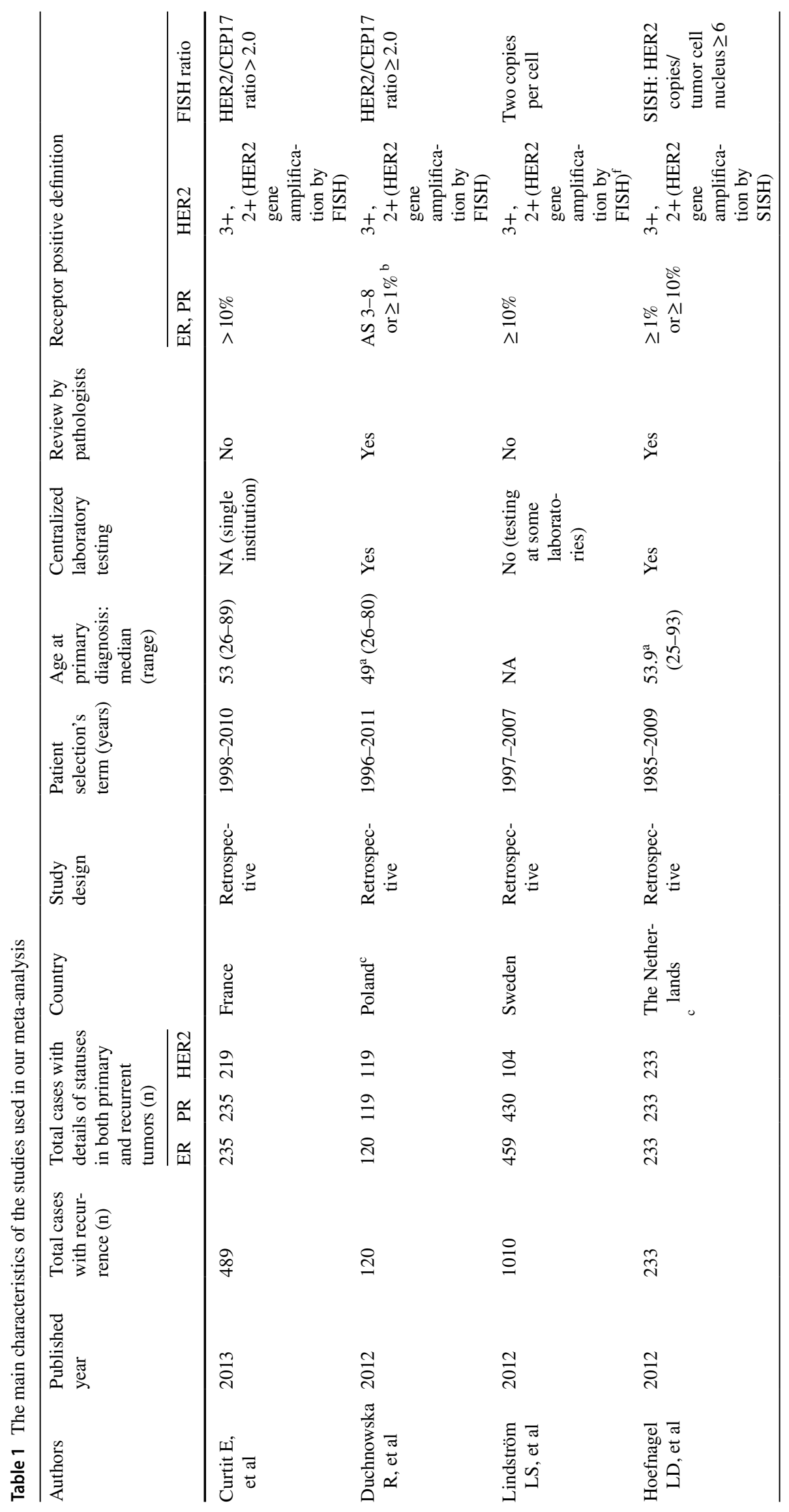




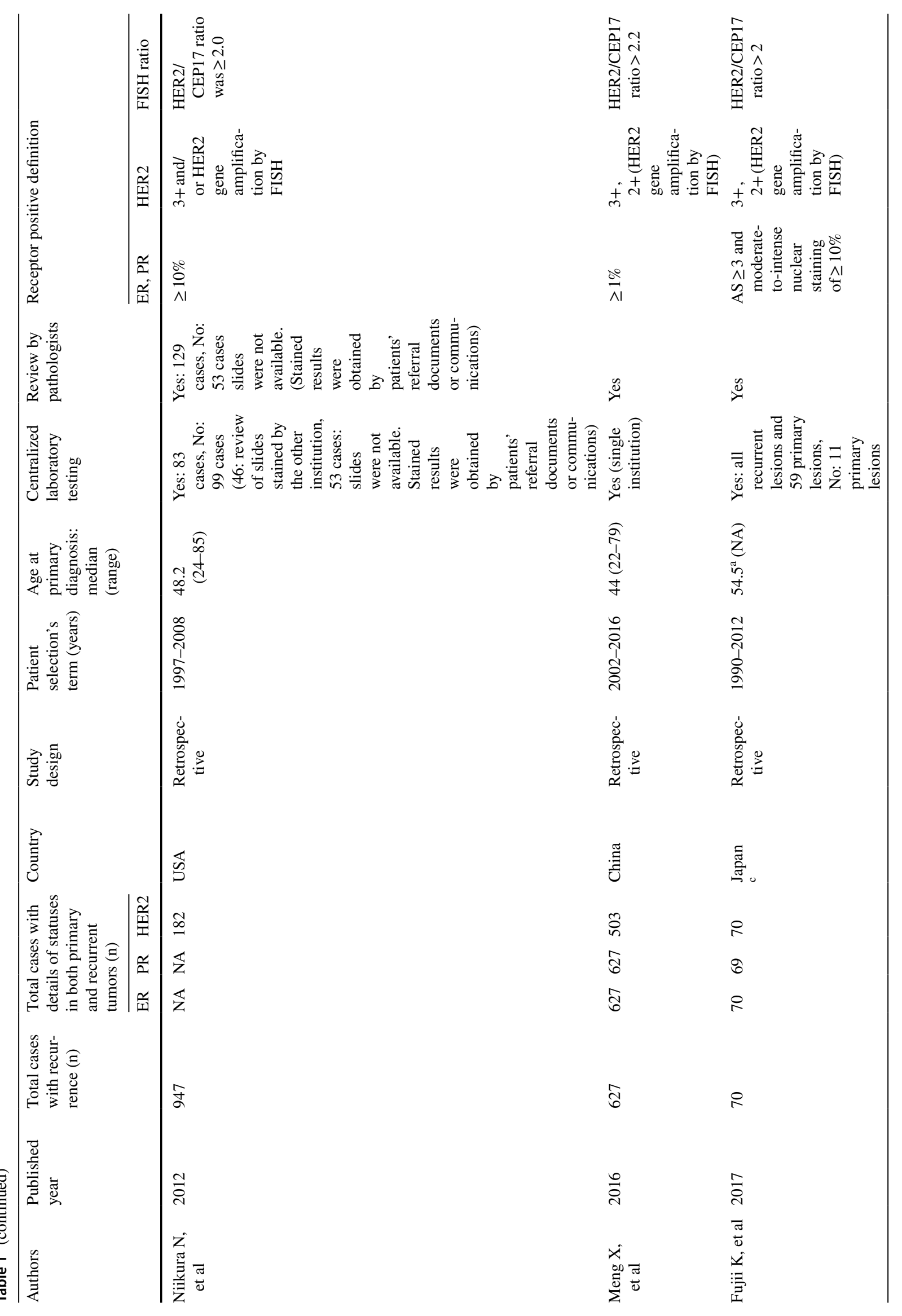




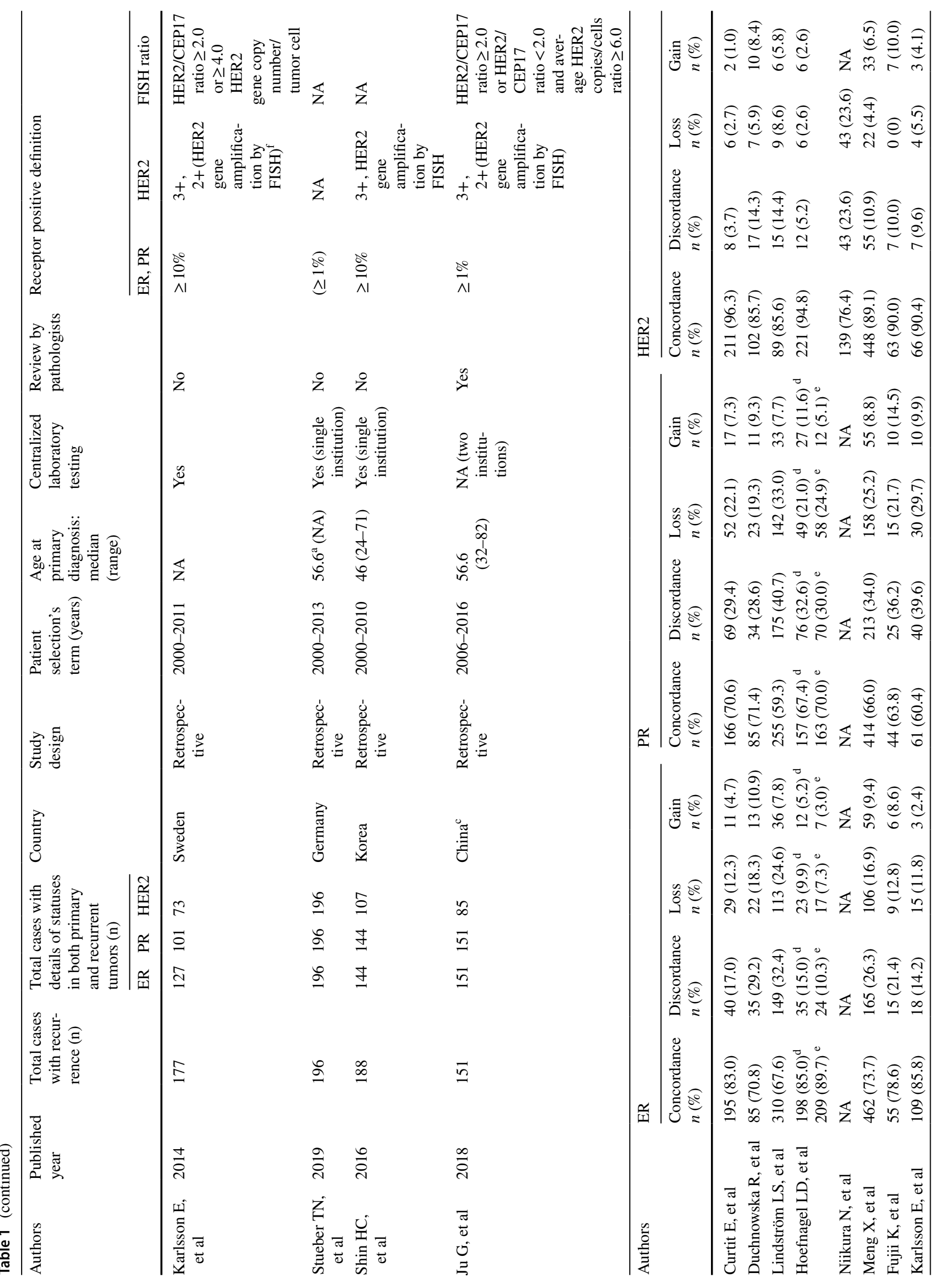


HR, 1.70; 95\% CI 1.34-2.15; $p<0.0001$; PR groups: HR, $1.49 ; 95 \%$ CI 1.20-1.86; $p=0.0003$ ) (Fig. 2A, C).

The ER-gain $(-/+)$ and PR-gain $(-/+)$ groups were not significantly associated with OS when compared with the ER-concordant (-/-) and PR-concordant (-/-) groups ([Fixed-effects model] ER groups: HR, 0.90; 95\% CI $0.68-1.19 ; p=0.45$; PR groups: HR, $0.77 ; 95 \%$ CI $0.59-1.01 ; p=0.06$, [Random-effects model] ER groups: HR, 0.98; 95\% CI 0.59-1.63; $p=0.94$; PR groups: HR, 0.81; 95\% CI 0.54-1.23; $p=0.32$ ) (Fig. 2B and D).

Data regarding HER2-loss analysis were available in only $2 / 11$ studies [27, 32]. However, these data were insufficient to perform a meta-analysis according to our methods. Likewise, data regarding HER2-gain analysis were also available in $2 / 11$ studies $[27,32]$. We were able to extract HR data from only one study according to our methods (HR, 2.17; 95\% CI 0.82-5.74; $p=0.12$ ) [27].

\section{Influence of receptor discordance on PRS}

The ER-loss (+/-) and PR-loss (+/-) groups were significantly associated with shorter PRS compared with the ERconcordant (+/+) and PR-concordant (+/+) groups, respectively ([Fixed-effects model] ER groups: HR, 1.72; 95\% CI 1.40-2.11; $p<0.00001 ;$ PR groups: HR, $1.54 ; 95 \%$ CI $1.27-1.87 ; p<0.00001$, [Random-effects model] ER groups: HR, 1.76; 95\% CI 1.39-2.22; $p<0.00001$; PR groups: HR, 1.56 ; 95\% CI 1.26-1.93; $p<0.0001$ ) (Fig. 3A and C).

The ER-gain $(-/+)$ group was significantly associated with longer PRS compared with the ER-concordant (-/-) group ([Fixed-effects model] HR, 0.76; 95\% CI 0.59-0.97; $p=0.03$, [Random-effects model] HR, 0.76; 95\% CI $0.59-0.97 ; p=0.03$ ) (Fig. 3B). There was no significant difference in PRS between the PR-gain $(-/+)$ and PR-concordant (-/-) groups ([Fixed-effects model] HR, 1.10; 95\% CI 0.85-1.43; $p=0.47$, [Random-effects model] HR, 1.12; 95\% CI 0.84-1.49; $p=0.45$ ) (Fig. 3D).

HER2-loss data were available in 3/11 studies [26, 28, 33 . Only one study was eligible for comparison between these two groups (HR, 2.78; 95\% CI 1.61-4.76; $p=0.0002$ ) [28]. HER2-gain data were available in two studies [26, 33], but the data were insufficient for meta-analysis.

\section{Influence on survival in patients with distant metastasis}

In the subgroup with only distant metastasis not including local recurrences, the OS of both ER-loss $(+/-)$ and PRloss $(+/-)$ groups was significantly shorter than those of ER-concordant $(+/+)$ and PR-concordant $(+/+)$ groups, respectively ([Fixed-effects model] ER groups: HR, 1.70; 95\% CI 1.35-2.14; $p<0.00001$; PR groups: HR, $1.28 ; 95 \%$ CI 1.02-1.60; $p=0.03$, [Random-effects model] ER groups: 
A
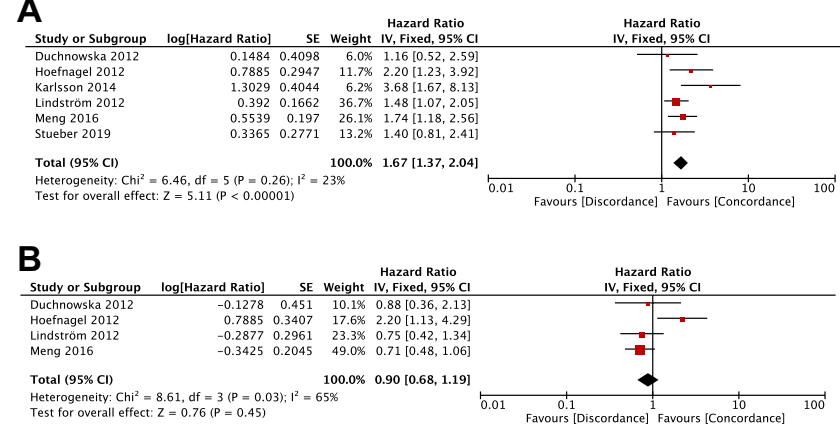

C

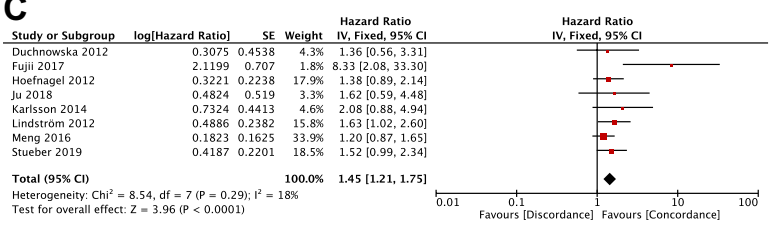

D

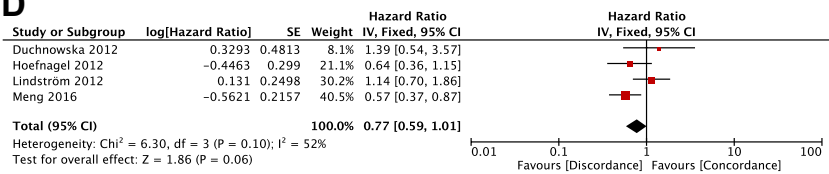

Fig. 2 Forest plots comparing overall survival between receptor-loss/ gain groups and receptor-concordant groups. A Comparison of OS between ER-loss $(+/-)$ group and ER-concordant $(+/+)$ group. B Comparison of OS between ER-gain $(-/+)$ group and ER-concordant
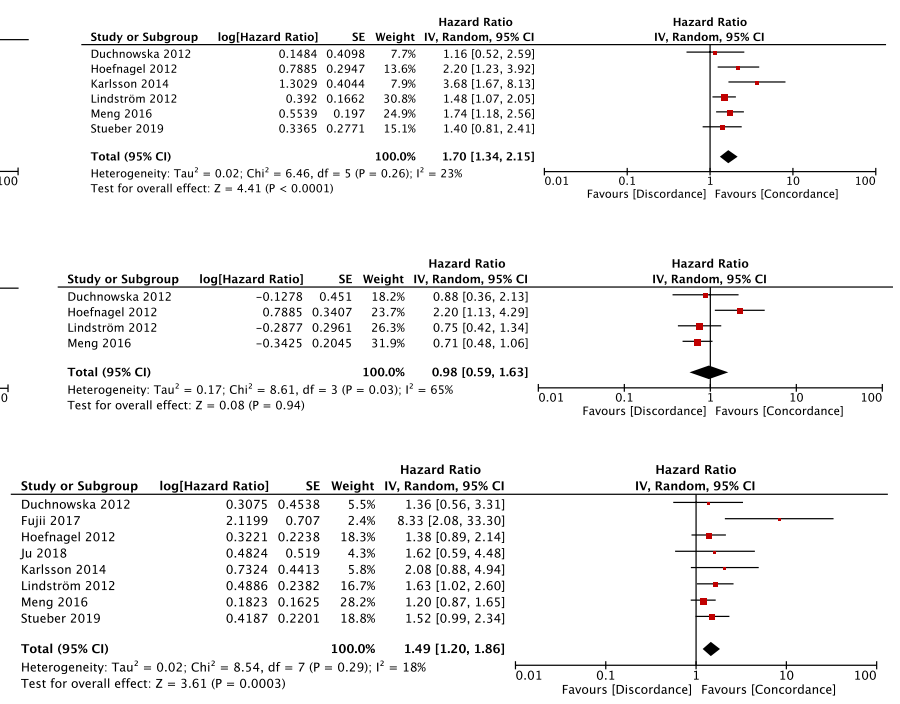

A
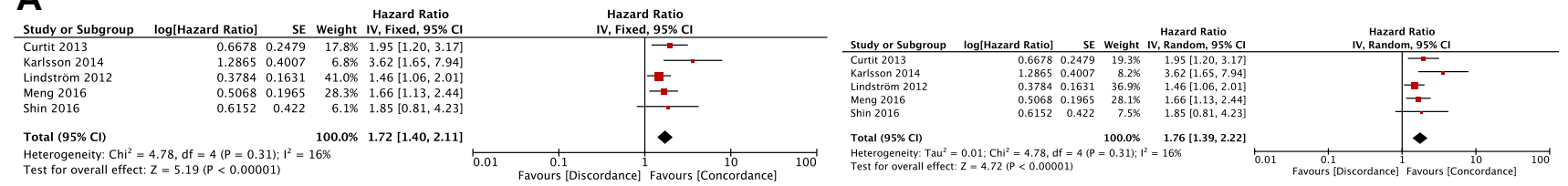

\section{B}

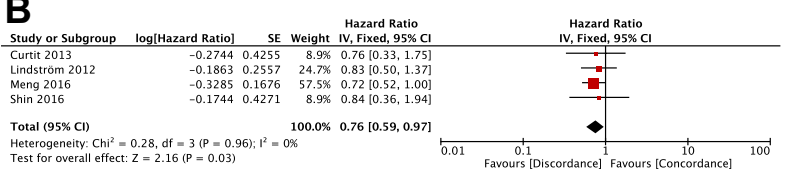

C

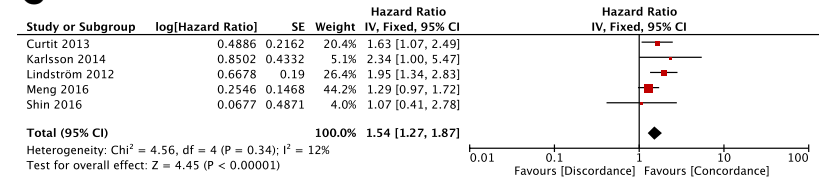

D

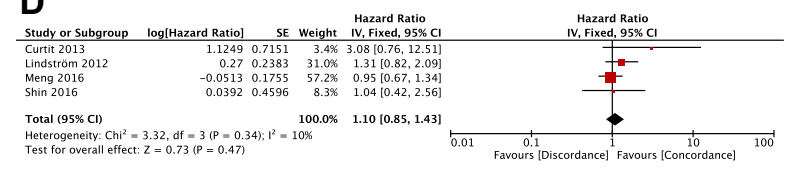

Fig. 3 Forest plots comparing post-recurrence survival between receptor-loss/gain group and receptor-concordant group. A Comparison of PRS between ER-loss (+/-) group and ER-concordant $(+/+)$ group. B Comparison of PRS between ER-gain (-/+) group and ER-concordant (-l-) group. C Comparison of PRS between PR-

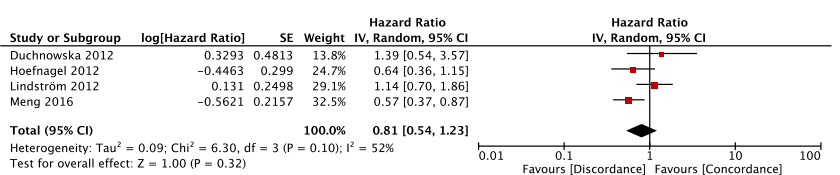

(-/-) group. C Comparison of OS between PR-loss (+/-) group and PR-concordant $(+/+)$ group. D Comparison of OS between PR-gain $(-/+)$ group and PR-concordant $(-/-)$ group. ER estrogen receptor, $O S$ overall survival, $P R$ progesterone receptor
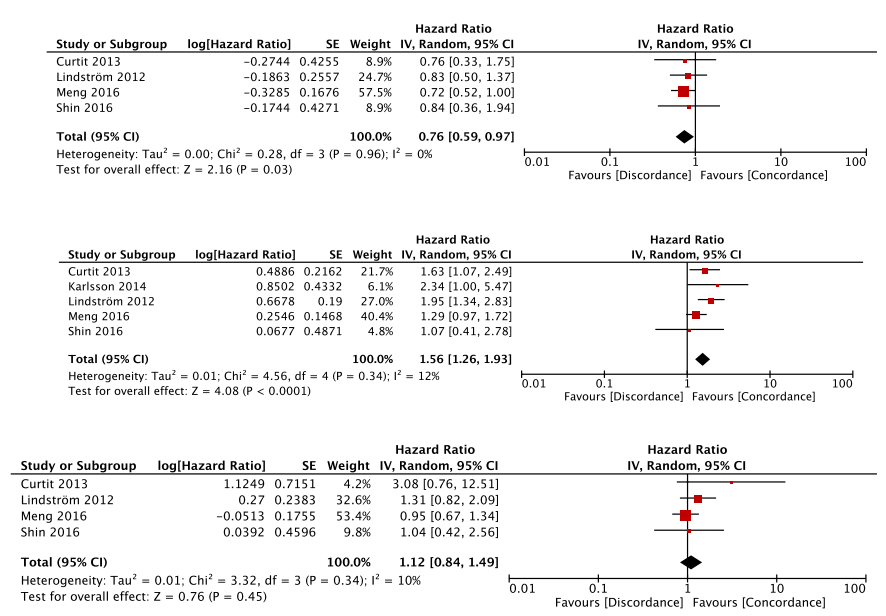

loss (+/-) group and PR-concordant (+/+) group. D Comparison of PRS between PR-gain (-/+) group and PR-concordant (-/-) group. $E R$ estrogen receptor, $P R$ progesterone receptor, $P R S$ post-recurrence survival 

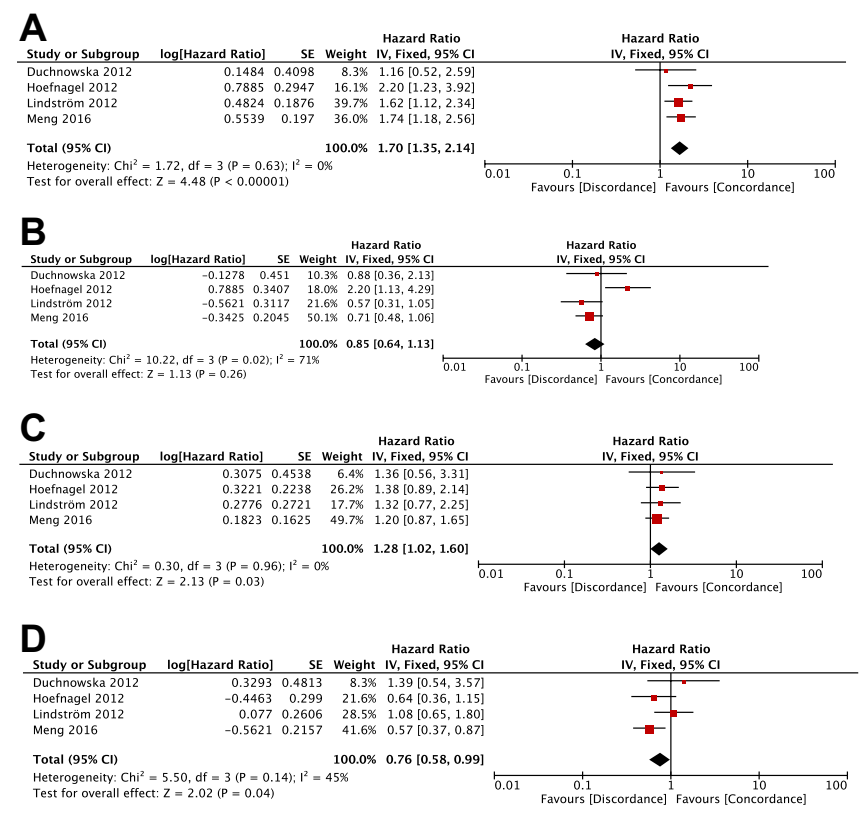

Fig. 4 Forest plots comparing overall survival between receptor-loss/ gain groups and receptor-concordant group in the subgroup of distant metastasis. A Comparison of OS between ER-loss $(+/-)$ group and ER-concordant $(+/+)$ group. B Comparison of OS between ER-gain $(-/+)$ group and ER-concordant $(-/-)$ group. C Comparison of OS

HR, 1.70; 95\% CI 1.35-2.14; $p<0.00001$; PR groups: HR, 1.28 ; 95\% CI 1.02-1.60; $p=0.03$ ) (Fig. 4A and C).

There was no significant difference in the OS between the ER-gain (-/+) and ER-concordant (-/-) groups ([Fixedeffects model] HR, $0.85 ; 95 \%$ CI $0.64-1.13 ; p=0.26$, [Random-effects model] HR, 0.92; 95\% CI 0.52-1.62; $p=0.77$ ) (Fig. 4B).

The PR-gain $(-/+)$ group was significantly associated with longer OS compared with the PR-concordant (-/-) group (HR, 0.76; 95\% CI 0.58-0.99; $p=0.04$ ) in the fixedeffects model but was not significant in the random-effects model (HR, 0.79; 95\% CI 0.54-1.17; $p=0.24$ ) (Fig. 4D).

\section{Subgroup analysis according to the threshold ( $1 \%$ or $10 \%$ ) of hormone receptor positivity}

The ER-loss (+/-) and PR-loss (+/-) groups were significantly associated with shorter OS compared with the ERconcordant $(+/+)$ and PR-concordant (+/+) group regardless of the definition of hormone receptor positivity and type of model (Supplementary Fig. S3A and S3C).

In subgroup analysis for the $1 \%$ and $10 \%$ thresholds, the ER-gain (-/+) group was not associated with OS compared with the ER-concordant $(-/-)$ group in both of the models, respectively (Supplementary Fig. S3B). Meanwhile, in the subgroup analysis of $1 \%$ threshold, the OS of PRgain $(-/+)$ group was significantly longer than those of

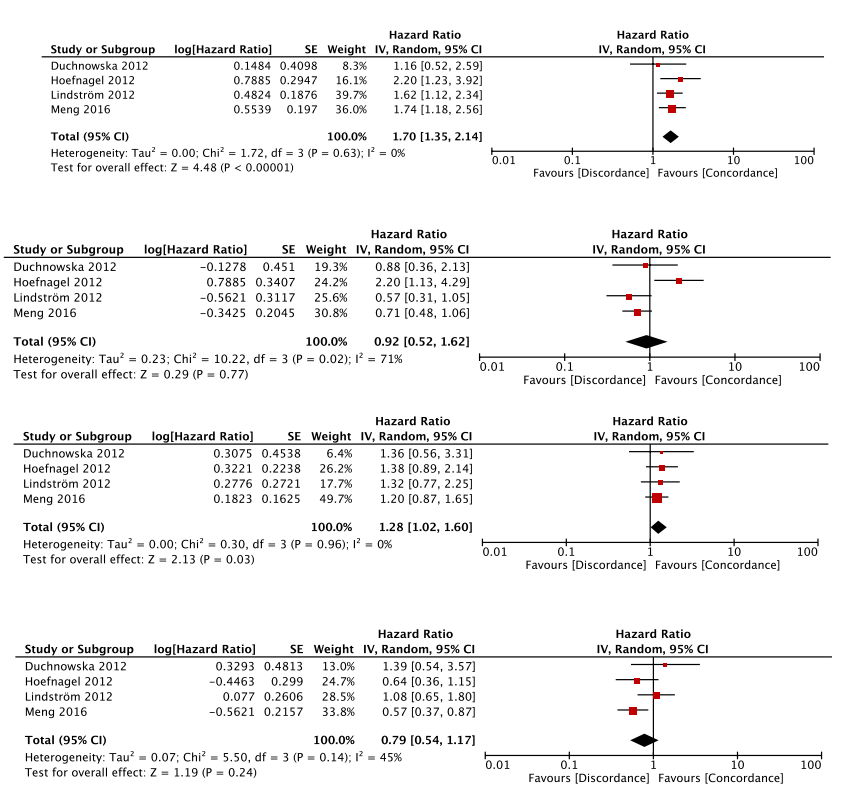

between PR-loss (+/-) group and PR-concordant (+/+) group. D Comparison of OS between PR-gain $(-/+)$ group and PR-concordant $(-/-)$ group. ER estrogen receptor, $O S$ overall survival, $P R$ progesterone receptor

the PR-concordant (-/-) group in the fixed-effects model (HR, 0.66; 95\% CI 0.47-0.90; $p=0.01$ ) but not significant in the random-effects model (HR, 0.68 ; $95 \%$ CI $0.45-1.03$; $p=0.07$ ) (Supplementary Fig. S3D).

There was no significant difference between the two subgroups in the ER-loss analysis ([Fixed-effects model] $I^{2}=0 \% ; p=0.83$, [Random-effects model] $I^{2}=0 \% ; p=0.81$ ). There was no significant difference between the two subgroups in the ER-gain analysis ([Fixed-effects model] $I^{2}=0 \% ; p=0.49$, [Random-effects model $] I^{2}=0 \% ; p=0.43$ ). Contrarily, there was a significant difference between the two subgroups in the PR-loss analysis ([Fixed-effects model] $I^{2}=81.8 \% ; p=0.02$, [Random-effects model] $I^{2}=77.2 \%$; $p=0.04)$. There was a significant difference between the two subgroups in the PR-gain analysis in the fixed-effects model $\left(I^{2}=79.0 \% ; p=0.03\right)$ but not in the random-effects model $\left(I^{2}=69.5 \% ; p=0.07\right)$.

\section{Subgroup analysis according to the assessment of risk of bias}

There was not a significant difference between the high- and low-risk-of-bias groups in the analysis of the OS in both models (Supplementary Fig. S4).

In the subgroup analysis of the low-risk-of-bias group, both the ER-loss (+/-) and PR-loss (+/-) groups had a significantly shorter OS than those of the ER-concordant 
$(+/+)$ and PR-concordant $(+/+)$ groups in the two models, respectively. Meanwhile, in the subgroup analysis of the low-risk-of-bias group, the PR-gain (-/+) group had a significantly longer OS than the PR-concordant (-/-) group in the fixed-effects model (HR, 0.74; 95\% CI 0.56-0.97; $p=0.03)$, but there was not a significant difference in the random-effects model (HR, $0.74 ; 95 \%$ CI $0.48-1.15$; $p=0.19$ ).

\section{Subgroup analysis according to the study area}

There was no significant difference between the Western group and Asian group in the receptor-loss analysis ([Fixed-effects model] ER-loss: $I^{2}=0 \% ; p=0.82$, PRloss: $I^{2}=0 \% ; p=0.51$, [Random-effects model] ER-loss: $I^{2}=0 \% ; p=0.96$, PR-loss: $I^{2}=0 \% ; p=0.53$; Supplementary Fig. S5).

In the receptor-gain analysis, there was no significant difference between the two subgroups ([Fixed-effects model] ER-gain: $I^{2}=61.0 \% ; p=0.11$, PR-gain: $I^{2}=70.5 \% ; p=0.07$, [Random-effects model] ER-gain: $I^{2}=23.3 \% ; p=0.25$, PRgain: $I^{2}=63.9 \% ; p=0.10$ ). Statistical heterogeneity was high in the PR-gain analysis in the two models.

\section{Evaluation of adjuvant therapy and changing treatment based on the status of each receptor}

In the 11 selected articles, the median rate of adjuvant therapy was observed in $42.9 \%$ (range $25.8-84.1 \%$ ), $57.9 \%$ (range 18.1-92.5\%), and 8.1\% (range 2.9-41.8\%) for hormonal therapy, chemotherapy, and HER2-targeted therapy, respectively (Supplementary Table S4). Of the 11 studies analyzed herein, adjuvant therapy could affect receptor discordance as follows: hormonal therapy and/or chemotherapy, 5/11 studies (45.5\%); HER2-targeted therapy, 1/11 studies (9.1\%). In the selected articles, we could not evaluate the changing rate of treatment according to receptor conversion in recurrent tumors due to insufficient data.

\section{Evaluation of heterogeneity and publication bias}

Statistical heterogeneity was mainly low to moderate in the total analysis of receptor-loss groups but was relatively moderate to high in the total analysis of receptor-gain groups. Particularly, heterogeneity in the ER-gain analysis on OS was significantly observed (Fig. 2B). One study showed poor survival in ER-gain groups with recurrent tumors [24], whereas the other three studies showed the tendency of better survival $[25,27,29]$. Thus, a meta-analysis was conducted in cases excluding the discrepant study, an analysis defined as "ER-gain' (-/+) group vs. ER-concordant' (-/-) group" (Supplementary Table S6). Statistical heterogeneity was not observed $\left(I^{2}=0 \% ; p=0.91\right)$, and the OS of the ERgain' $(-/+)$ group was marginally associated with better OS than that of the ER-concordant' (-I-) group (HR, 0.74; 95\% CI $0.54-1.01 ; p=0.06)$.

Meanwhile, in the PR-gain analysis on OS, statistical heterogeneity was marginally high (Fig. 2D). We performed meta-regression analysis to investigate whether the HR of PR-gain $(-/+)$ could influence the rates of the other variables in the primary tumors for each article. The rate of HER2-positivity in the primary tumor was significantly correlated with the HR of PR-gain $(-/+)$ on the OS analysis $(p=0.034)$ (Supplementary Table S7 and Supplementary Fig. S6).

We were unable to evaluate the risks of publication bias with Egger's test because each analysis contained fewer than ten studies. Funnel plots on each analysis are summarized in Supplementary Fig. S7.

\section{Discussion}

In the current meta-analysis study, we showed that loss of either ER or PR in recurrent tumors was significantly associated with poor prognosis in terms of OS and PRS. Gain of ER tends to be associated with better outcome in terms of longer PRS but not OS compared with receptor-negative primary and recurrent tumors. HER2 loss and gain could be related to poor outcome as compared with receptor-positive and receptor-negative concordance, respectively. As Li et al. reported a meta-analysis on the prognostic impact of receptor discordance after neoadjuvant chemotherapy, it did not investigate patient survival in cases of recurrent tumors [35]. To our knowledge, this is the first report to investigate whether receptor discordance affects the survival of patients with recurrent tumors using the meta-analytic method.

In the analysis of distant metastasis (Fig. 4), trends for each result were similar to the total analysis of OS (Fig. 2). Furthermore, statistical heterogeneity tended to be lower compared with those analyses. It is estimated that there are survival differences between local and distant in some previous reports [36, 37]. However, the reason for this could not be clarified herein because survival differences of patients that had only local recurrence not having distant metastases between receptor discordance and receptor concordance were unavailable.

In our meta-analysis, we found that significant heterogeneity between the $1 \%$ and $10 \%$ thresholds was found during PR analysis. The reason for statistical heterogeneity has not been clarified; however, previous studies have suggested that the rate of PR positivity is a more continuous variable as a prognostic factor [15, 38]. In particular, some studies have reported that cases with 1-10\% PR expression behaved the same as those with receptor-negative disease [39-41]. Thus, 
the prognosis would be different according to the threshold definition.

Some studies have reported that adjuvant therapy could influence the receptor discordance in our selected articles. However, because our meta-analysis did not focus it as a primary endpoint, the relation between receptor discordance and adjuvant treatment requires further investigation. Meanwhile, some studies have reported a treatment strategy that was altered in reference to receptor changes in recurrent BC [1, 2, 42-44]. Moreover, discordant cases could lead to inappropriate hormone therapy treatments after the recurrence $[2,10]$ and treatment alteration based on receptor discordance could affect patient survival [29]. Those findings and our results highly recommended performing a biopsy of recurrent tumors to provide information for altering treatment strategies. However, performing a biopsy of all recurrent sites is invasive, costly, and cannot describe all recurrent sites. Liquid biopsy may be beneficial to detect tumor heterogeneity in circulating tumor cells $[45,46]$.

Discordance in those receptors has been reported due to analytical error, intratumoral heterogeneity [47], and cellular clonal evolution $[48,49]$. The mechanisms of receptor discordance have been gradually elucidated [50]. Unfortunately, the molecular mechanisms for receptor discordance cannot be explained by the results of our study.

Some limitations of our study are as follows: (1) We could not perform a meta-analysis for HER 2 discordance. The total number of HER2-discordant cases was too small in each study. A large number of studies will be required to verify these findings, especially for the HER2-gain group. Additionally, all studies were mainly based on 2007 or 2013 ASCO/CAP guidelines [17, 18] but not on the updated guidelines [51]. (2) In cases that we were not able to get $\mathrm{HR}$ and $95 \% \mathrm{CI}$ data even if we inquired each corresponding author to get the data about HR and 95\% CI on each article, we extracted those data using specialized software. This technique is commonly used to perform meta-analyses, but the calculated data are not the same as the original data, especially in cases of including more censored cases as most studies include censored data. Additionally, one article suggested no significant differences, but our calculations showed a significant association with poor survival [24]. (3) Each cohort contained different clinicopathological characteristics and different treatment options (adjuvant therapies or systemic therapies after recurrence), particularly between hormone receptor-positive and negative patients. Multivariate analytic results to adjust the confounding factors were mentioned in some original articles but not all articles. Thus, our conclusion regarding the effect of receptor discordance may not be robust as clinicopathological characteristics and treatment options are different among each cohort and our analysis could not fully adjust the confounding factors. In cases wherein clinicopathological characteristics are different among studies, a random-effects model results could be more appropriate than a fixed-effects model. Lim et al. have reported that the clinicopathological staging and survival outcomes are different between Asian and European groups [52]. Therefore, a random-effects model results would be more recommended in the subgroup analysis according to the study area. (4) Our meta-analysis included many studies performed over several decades, which could introduce bias because treatment strategies could vary with time.

In conclusion, in this meta-analysis, we have shown that receptor discordance between primary tumors and recurrent tumors could be associated with patient OS and PRS compared with receptor concordance. Analyzing receptor status to confirm the diagnosis of recurrent $\mathrm{BC}$ would provide information for altering treatment strategies based on either receptor-loss or receptor-gain status of recurrent tumors.

Supplementary Information The online version contains supplementary material available at https://doi.org/10.1007/s10549-021-06390-6.

Acknowledgements We would like to thank Alison Ashmore (University of Nottingham Libraries) for support to construct searching strategies; Naoki Niikura M.D., Ph.D. and Naoto Ueno M.D., Ph.D., F.A.C.P. for providing additional data; Kimihito Fujii M.D., Ph.D. for providing additional data. We would like to thank Enago (www.enago. jp) for the English language review.

Author contributions SS, GB, and EAR conceptualized and designed the study. SS, BMS, and SK collected articles and evaluated those data. SS and GB performed the statistical analysis and interpreted the data. SS, GB, ARG, HT, ST, AS, and EAR wrote, reviewed, and revised the manuscript. All authors approved the final manuscript as submitted and agreed to be accountable for all aspects of the work.

Funding This study was supported in part by Japan Society for the Promotion of Science (JSPS) Overseas Challenge Program for Young Researchers.

\section{Declarations}

Conflict of interest SK has received honoraria from Daiichi Sankyo co. ltd, Taiho Pharmaceutical Co. Ltd, Eli Lilly and Company, MSD K.K., AstraZeneca K.K., and Novartis Japan. HT received research grant (Taiho Pharmaceutical Co. Ltd., GORYO Chemical, Inc., and Roche Diagnostics K.K.) and scholarship donation (Chugai Pharmaceutical Co. Ltd., Takeda Pharmaceutical Co. Ltd., and Eli Lilly and Company). The other authors declare no conflict of interest.

Ethics approval and consent to participate This study used only previous published data and does not include original participants' data.

Open Access This article is licensed under a Creative Commons Attribution 4.0 International License, which permits use, sharing, adaptation, distribution and reproduction in any medium or format, as long as you give appropriate credit to the original author(s) and the source, provide a link to the Creative Commons licence, and indicate if changes were made. The images or other third party material in this article are included in the article's Creative Commons licence, unless indicated 
otherwise in a credit line to the material. If material is not included in the article's Creative Commons licence and your intended use is not permitted by statutory regulation or exceeds the permitted use, you will need to obtain permission directly from the copyright holder. To view a copy of this licence, visit http://creativecommons.org/licenses/by/4.0/.

\section{References}

1. Thompson AM, Jordan LB, Quinlan P et al (2010) Prospective comparison of switches in biomarker status between primary and recurrent breast cancer: the Breast Recurrence In Tissues Study (BRITS). Breast Cancer Res 12:R92

2. Amir E, Miller N, Geddie W et al (2012) Prospective study evaluating the impact of tissue confirmation of metastatic disease in patients with breast cancer. J Clin Oncol 30:587-592

3. Lower EE, Glass E, Blau R et al (2009) HER-2/neu expression in primary and metastatic breast cancer. Breast Cancer Res Treat 113:301-306

4. Hoefnagel LD, van der Groep P, van de Vijver MJ et al (2013) Discordance in ER $\alpha, \mathrm{PR}$ and HER2 receptor status across different distant breast cancer metastases within the same patient. Ann Oncol 24:3017-3023

5. Schrijver WAME, Suijkerbuijk KPM, Van Gils CH et al (2018) Receptor conversion in distant breast cancer metastases: a systematic review and meta-analysis. J Natl Cancer Inst 110:568-580

6. Aurilio G, Disalvatore D, Pruneri G et al (2014) A meta-analysis of oestrogen receptor, progesterone receptor and human epidermal growth factor receptor 2 discordance between primary breast cancer and metastases. Eur J Cancer 50:277-289

7. Van Poznak C, Somerfield MR, Bast RC et al (2015) Use of biomarkers to guide decisions on systemic therapy for women with metastatic breast cancer: American society of clinical oncology clinical practice guideline. J Clin Oncol 33:2695-2704

8. NCCN clinical practice guidelines in oncology: breast cancer. National Comprehensive Cancer Network. https://www.nccn.org/ store/login/login.aspx?ReturnURL=https://www.nccn.org/profe ssionals/physician_gls/pdf/breast.pdf

9. Turner NH, Di Leo A (2013) HER2 discordance between primary and metastatic breast cancer: assessing the clinical impact. Cancer Treat Rev 39:947-957

10. Liedtke C, Broglio K, Moulder S et al (2009) Prognostic impact of discordance between triple-receptor measurements in primary and recurrent breast cancer. Ann Oncol 20:1953-1958

11. Dieci MV, Barbieri E, Piacentini F et al (2013) Discordance in receptor status between primary and recurrent breast cancer has a prognostic impact: A single-institution analysis. Ann Oncol 24:101-108

12. Moher D, Shamseer L, Clarke M et al (2015) Preferred reporting items for systematic reviews and meta-analysis protocols (PRISMA-P) 2015 statement. Syst Rev 4:1

13. Union for International Cancer Control. TNM Classification of malignant tumours. Eighth edition. (2017)

14. Senkus E, Kyriakides S, Ohno S et al (2015) Primary breast cancer: ESMO clinical practice guidelines for diagnosis, treatment and follow-up. Ann Oncol 26:v8-30

15. Allison KH, Hammond MEH, Dowsett M et al (2020) Estrogen and progesterone receptor testing in breast cancer: ASCO/CAP guideline update. J Clin Oncol 38:1346-1366

16. Hammond MEH, Hayes DF, Dowsett M et al (2010) American Society of Clinical Oncology/College of American Pathologists guideline recommendations for immunohistochemical testing of estrogen and progesterone receptors in breast cancer. J Clin Oncol 28:2784-2795

17. Wolff AC, Hammond MEH, Schwartz JN et al (2007) American Society of Clinical Oncology/College of American Pathologists guideline recommendations for human epidermal growth factor receptor 2 testing in breast cancer. J Clin Oncol 25:118-145

18. Wolff AC, Hammond MEH, Hicks DG et al (2013) Recommendations for human epidermal growth factor receptor 2 testing in breast cancer: American Society of Clinical Oncology/College of American Pathologists clinical practice guideline update. J Clin Oncol 31:3997-4013

19. Review Manager (RevMan) [Computer program]. Version 5.3. Copenhagen: The Nordic Cochrane Centre, The Cochrane Collaboration (2014)

20. Tierney JF, Stewart LA, Ghersi D et al (2007) Practical methods for incorporating summary time-to-event data into meta-analysis. Trials 8:16

21. Yang YF, Liao YY, Yang M et al (2014) Discordances in ER, PR and HER2 receptors between primary and recurrent/metastatic lesions and their impact on survival in breast cancer patients. Med Oncol 31:214

22. Ibrahim T, Farolfi A, Scarpi E et al (2013) Hormonal receptor, human epidermal growth factor receptor-2, and Ki67 discordance between primary breast cancer and paired metastases: clinical impact. Oncology 84:150-157

23. Lower EE, Glass EL, Bradley DA et al (2005) Impact of metastatic estrogen receptor and progesterone receptor status on survival. Breast Cancer Res Treat 90:65-70

24. Hoefnagel LD, Moelans CB, Meijer SL et al (2012) Prognostic value of estrogen receptor $\alpha$ and progesterone receptor conversion in distant breast cancer metastases. Cancer 118:4929-4935

25. Lindström LS, Karlsson E, Wilking UM et al (2012) Clinically used breast cancer markers such as estrogen receptor, progesterone receptor, and human epidermal growth factor receptor 2 are unstable throughout tumor progression. J Clin Oncol 30:2601-2608

26. Curtit E, Nerich V, Mansi L et al (2013) Discordances in estrogen receptor status, progesterone receptor status, and HER2 status between primary breast cancer and metastasis. Oncologist 18:667-674

27. Duchnowska R, Dziadziuszko R, Trojanowski T et al (2012) Conversion of epidermal growth factor receptor 2 and hormone receptor expression in breast cancer metastases to the brain. Breast Cancer Res 14:R119

28. Niikura N, Liu J, Hayashi N et al (2012) Loss of human epidermal growth factor receptor 2 (HER2) expression in metastatic sites of HER2-overexpressing primary breast tumors. J Clin Oncol 30:593-599

29. Meng X, Song S, Jiang ZF et al (2016) Receptor conversion in metastatic breast cancer: A prognosticator of survival. Oncotarget 7:71887-71903

30. Fujii K, Watanabe R, Ando T et al (2017) Alterations in three biomarkers (Estrogen receptor, progesterone receptor and human epidermal growth factor 2) and the Ki67 index between primary and metastatic breast cancer lesions. Biomed Rep 7:535-542

31. Karlsson E, Appelgren J, Solterbeck A et al (2014) Breast cancer during follow-up and progression - A population based cohort on new cancers and changed biology. Eur J Cancer 50:2916-2924

32. Stueber TN, Weiss CR, Woeckel A et al (2019) Influences of adjuvant treatments in hormone receptor positive breast cancer on receptor conversion in recurrent breast cancer. Arch Gynecol Obstet 299:533-541

33. Shin HC, Han W, Moon HG et al (2016) Patients with concordant triple-negative phenotype between primary breast cancers and corresponding metastases have poor prognosis. J Breast Cancer $19: 268-274$ 
34. Ju G, Zhu R, Zhao H et al (2018) The discordance pattern of molecular sub-types between primary and metastatic sites in Chinese breast cancer patients. Int J Clin Exp Pathol 11:5938-5947

35. Li C, Fan H, Xiang Q et al (2019) Prognostic value of receptor status conversion following neoadjuvant chemotherapy in breast cancer patients: a systematic review and meta-analysis. Breast Cancer Res Treat 178:497-504

36. Curcio LD, Chu DZ, Ahn C et al (1997) Local recurrence in breast cancer: Implications for systemic disease. Ann Surg Oncol 4:24-27

37. Lê MG, Arriagada R, Spielmann M et al (2002) Prognostic factors for death after an isolated local recurrence in patients with earlystage breast carcinoma. Cancer 94:2813-2820

38. Dowsett M, Allred C, Knox J et al (2008) Relationship between quantitative estrogen and progesterone receptor expression and human epidermal growth factor receptor 2 (HER-2) status with recurrence in the arimidex, tamoxifen, alone or in combination trial. J Clin Oncol 26:1059-1065

39. Raghav KPS, Hernandez-Aya LF, Lei X et al (2012) Impact of low estrogen/progesterone receptor expression on survival outcomes in breast cancers previously classified as triple negative breast cancers. Cancer 118:1498-1506

40. Chen T, Zhang N, Moran MS et al (2018) Borderline ER-positive primary breast cancer gains no significant survival benefit from endocrine therapy: A systematic review and meta-analysis. Clin Breast Cancer 18:1-8

41. Balduzzi A, Bagnardi V, Rotmensz N et al (2014) Survival outcomes in breast cancer patients with low estrogen/progesterone receptor expression. Clin Breast Cancer 14:258-264

42. Amir E, Clemons M, Purdie CA et al (2012) Tissue confirmation of disease recurrence in breast cancer patients: pooled analysis of multi-centre, multi-disciplinary prospective studies. Cancer Treat Rev 38:708-714

43. Simmons C, Miller N, Geddie W et al (2009) Does confirmatory tumor biopsy alter the management of breast cancer patients with distant metastases? Ann Oncol 20:1499-1504
44. Curigliano G, Bagnardi V, Viale G et al (2011) Should liver metastases of breast cancer be biopsied to improve treatment choice? Ann Oncol 22:2227-2233

45. Venesio T, Siravegna G, Bardelli A et al (2018) Liquid biopsies for monitoring temporal genomic heterogeneity in breast and colon cancers. Pathobiology 85:146-154

46. Criscitiello C, André F, Thompson AM et al (2014) Biopsy confirmation of metastatic sites in breast cancer patients: clinical impact and future perspectives. Breast Cancer Res 16:205

47. Campbell LL, Polyak K, (2007) Breast tumor heterogeneity: cancer stem cells or clonal evolution? Cell Cycle 6:2332-2338

48. Fisher R, Pusztai L, Swanton C (2013) Cancer heterogeneity: Implications for targeted therapeutics. Br J Cancer 108:479-485

49. Gerlinger M, Rowan AJ, Horswell S et al (2012) Intratumor heterogeneity and branched evolution revealed by multiregion sequencing. N Engl J Med 366:883-892

50. Yates LR, Knappskog S, Wedge D et al (2017) Genomic evolution of breast cancer metastasis and relapse. Cancer Cell 32:169-184. e7

51. Wolff AC, Hammond MEH, Allison KH et al (2018) Human epidermal growth factor receptor 2 testing in breast cancer: American society of clinical oncology/college of American pathologists clinical practice guideline focused update. J Clin Oncol 36:2105-2122

52. Lim DW, Giannakeas V, Narod SA (2020) Survival differences in Chinese versus white women with breast cancer in the United States: A SEER-Based Analysis. JCO Glob Oncol 6:1582-1592

Publisher's Note Springer Nature remains neutral with regard to jurisdictional claims in published maps and institutional affiliations. 